\title{
When (Not) to Rely on the Reliable Change Index
}

\author{
Andrew A. McAleavey \\ Center for Health Research, Helse Førde \\ Department of Psychiatry, Weill Cornell Medical College
}

16 December 2022. Subject to change.

All supplementary materials can be accessed here:

https://osf.io/rtg9k/?view only=68f0a247940f417aa3e0ab9ff522697e.

\section{Author Note}

Andrew A. McAleavey (iD https://orcid.org/0000-0001-5986-2033

I have no conflict of interest to disclose.

Correspondence concerning this manuscript should be addressed to Andrew A. McAleavey, Helse Førde, Svanehaugvegen 2, 6812 Førde, Norway. Email: andrew.athan.mcaleavey@helse-forde.no 


\begin{abstract}
The reliable change index $(\mathrm{RCI})$ is a widely used statistical tool to account for measurement error when evaluating difference scores. Because of its conceptual and computational simplicity, it has become widespread in research and applied psychology. However, researchers have repeatedly demonstrated ways that the RCI is insufficient or invalid for various applications. The aims of this manuscript are to describe the formulation and assumptions of the RCI, to summarize the rationale justifying the RCI, and to identify what is needed for proper calculation of the RCI when it is used. It is apparent that the RCI is the best available method only in a small number of situations, is easily miscalculated, and produces incorrect inferences more often than alternatives, largely because it is highly insensitive to real changes. It is argued that the RCI effectively discourages the collection of appropriate data, and introduces error rather than accounts for it on the whole. Many suggested alternatives are provided to help researchers and applied users. While the RCI has a clear rationale and some appropriate uses, it fails to address the central issue at hand: two observations of a noisy measure are insufficient data.
\end{abstract}

Keywords: Reliable Change Index (RCI); measurement error; clinical significance; reliable and clinically significant change; reliable trend index (RTI)

Public health significance statement: The reliable change index (RCI) is a statistical method used widely in psychology and medicine, yet there remains disagreement about its application. This manuscript summarizes the concepts involved in the RCI, shows when it may be applied validly, and demonstrates its insensitivity to real changes. Alternative methods for detecting reliable changes are demonstrated. 


\section{When (Not) to Rely on the Reliable Change Index}

The reliable change index $(\mathrm{RCI})$ is a common method for evaluating the statistical reliability of a difference score between two observations from the same individual. Named the "RC index" by Jacobson et al. (1984), revised by Christensen and Mendoza (1986), it is commonly attributed to Jacobson \& Truax (1991). In this context, the RCI was half of a two-part clinical significance test, though it is sometimes applied as an independent test of real or meaningful difference. It is worth noting that the RCI had been identified for some time prior to Jacobson et al.'s 1984 paper giving it that name. As detailed in Maassen (2000), the RCI is a repackaging of more formative classical test theory from the middle of the $20^{\text {th }}$ century (see, e.g., McNemar, 1958). It is not surprising that the same formula is used widely, sometimes under different names (e.g., in Bland \& Altman, 1996). However, perhaps because of the Jacobson papers' clinical focus, the RCI is now pervasive in psychology. The 1991 paper has been cited over 10,000 times according to Google Scholar, across disciplines of medicine and social science. PubMed documents increases in citations to this manuscript almost every year since its publication, and it was the most cited article in the history of the Journal of Consulting and Clinical Psychology thirty years after its publication (American Psychological Association, 2021).

However, the RCI has also sparked a large volume of criticism. These critiques cover a wide

range, with authors sometimes refining its formula, identifying circumstances in which it is outperformed, and entirely rejecting its use in certain conditions. This critical literature is overwhelmingly neglected, ignored, or dismissed by applied researchers. This may be because alternatives appear difficult to implement (Jacobson et al., 1999; Ronk et al., 2016), alternatives may seem quite similar to the RCI (Atkins et al., 2005; Lassere et al., 2001), or an evaluation that the RCI remains acceptable, even if flawed (Blampied, 2022; Duff, 2012; Maassen, 2000, 2001; Speer \& Greenbaum, 2002).

The purpose of this manuscript is to offer guidance as to when the RCI is appropriate. The particular focus is mental health outcomes, especially patient-reported outcome measures (PROMs) for mental health symptoms, since the RCI seems to be particularly popular in this field.

\section{The RCI}


The "RC index" for an individual, as popularized by Jacobson and Truax (1991), is:

$$
R C=\frac{x_{2}-x_{1}}{\sqrt{2\left(s_{1} \sqrt{1-r_{x x}}\right)^{2}}}
$$

Where $x_{1}$ and $x_{2}$ are the observed outcomes for one individual at times 1 and $2 ; r_{x x}$ is a reliability coefficient; and $s_{1}$ is a standard deviation. The denominator of Eq. 1 is sometimes called the standard error of the difference score, or $\mathrm{S}_{\text {diff. }}$ As recommended by the authors, if the RC index is greater than 1.96, we reject the null hypothesis that the true difference score is 0 with $95 \%$ confidence. In some subfields, it has become common to use 1.645 , reflecting $90 \%$ confidence (Duff, 2012). These reflect Type I error rates of .05 and .1 .

Alternatively, the instrument's RCI has become a common quantity as well:

$$
R C I=1.96 \times \sqrt{2\left(s_{1} \sqrt{1-r_{x x}}\right)^{2}}=1.96 \times \mathrm{S}_{\text {diff }}
$$

This change leads to some confusion but is identical to the original with 5\% Type I error. Much like a critical value, if an observed difference score is larger than the instrument's RCI, the difference is considered "reliable." In other contexts, Eq. 2 is referred to as the minimally detectable change (MDC), repeatability (Bland \& Altman, 1996), and other terms (Hays \& Peipert, 2021). The RCI for a scale will be larger when the instrument is not reliable and/or typical scores vary a great deal, which is logical.

Given its commonality, the term "RCI" in this manuscript refers to the instrument-focused Eq. 2 rather than the individual-focused Eq. 1. Do note, however, that this definition appears to place all the uncertainty on the instrument, rather than the observations. Instead of estimating how uncertain we are about a specific observed difference score, we declare that an observed difference is reliable or not; it is harder to see how reliable a given change is. This is a loss.

\section{Using the RCI means you have inadequate data}

Any time the RCI is used, it is used because the desired data has not been collected. An analogy may help illustrate this. Let us say that we have a 5-year-old child, Mac, who wants to know if he is growing taller. We measure his height two times, one month apart. At time 1, he was $98 \mathrm{~cm}$ tall. At time 2, 
he was $99 \mathrm{~cm}$. This seems to suggest that Mac grew $1 \mathrm{~cm}$, and he is thrilled. However, our measures were imperfect: we used a simple pencil marking system with a hand as a level, which obviously has some error. Each observation could be off by some distance, and the difference could be large just due to error. We don't know how large it could be though. Given this, what should we tell Mac? Did he grow?

The first - and by far most important - point to take from this example is that our data are insufficient. Mac has a question, and we collected some data, but this does not mean we have the right data to answer his question. More trustworthy measurement may be necessary to accurately detect growth over just one month. Without changing the measurement tool, we would need to see a very large change before we felt confident our errors were not responsible. Alternatively, we could have improved our data by collecting more observations of Mac, even with our imprecise method. For instance, if we had measured Mac's height several times on the first day with measurements of 97, 97, 97, 98, and 97cm, and several more times a month later, with $99,100,100,99,100 \mathrm{~cm}$ we would be confident that some growth had happened. Alternatively, if we had measured Mac twice a week for a month, with measurements of $98,99,98,99,98,99,98$, and $99 \mathrm{~cm}$, we would be likely to conclude that no (observable) change had occurred. However, all we have is our two noisy observations.

This is always the case when the RCI is used: we have not collected appropriate data. This is true even when we have observed a huge change. If we had observed $9 \mathrm{~cm}$ of growth in a month, we should be extremely skeptical about the accuracy of our measurement. It's more likely there is a huge error rather than such a huge change, and if our measures were at all accurate, we should have no uncertainty that Mac grew. We only have the bare minimum data to estimate change, and not enough data to estimate error. We do not know how insufficient the data are, just that it is not good enough for our purpose.

Clearly, we should collect better data, but the RCI allows us to avoid this. Rather than collecting better data, the basic strategy of the RCI is to first assume that our observed change is a good (unbiased) estimate of true change, then assume that the error is similar to errors seen in other cases. Given these assumptions, all we need is an estimate of error in similar cases. That is the RCI. To derive the RCI for Mac, we might measure the heights of all of Mac's classmates, using the same measurement technique. 
After a few minutes, we would measure them all again. We can then estimate how noisy our system is because any difference in the children's heights should be due to measurement error. Say that we find half the children are measured at the same height both times, and the other half are measured as $1 \mathrm{~cm}$ different. We calculate an estimate of a "typical" error in a change score $\left(\mathrm{S}_{\text {diff }}\right): 0.71 \mathrm{~cm}$. The RCI is an unusually large, but plausible, error. In this example, only a difference greater than $1.39 \mathrm{~cm}$ would be "reliable" using the RCI. Mac's observed change of $1 \mathrm{~cm}$ is not reliable. Perhaps Mac did not grow.

In this way, the RCI seems to solve a difficult problem: We have a question (Did Mac grow?), and some data $(98 \mathrm{~cm}$ and $99 \mathrm{~cm})$, but this data is insufficient. The RCI provides something like - but definitely not - data we wish we had.

\section{The RCI is one statistical tool among many}

The RCI is meant as a "purely statistical" (Jacobson et al., 1984, p. 344) test of "statistical reliability" (p. 347) used as part of a test of clinically significant change. Why do we need a statistical test? Measurement error. Under common statistical theories, observed scores are thought to comprise true score (which is of interest) and measurement error (which is not). As the authors state, the RCI "tells us whether change reflects more than the fluctuations of an imprecise measuring instrument" (Jacobson et al., 1984, p. 344; Jacobson \& Truax, 1991, p. 14). More technically, the RCI quantifies our certainty that two observed scores reflect the same true score, since we might have different observations if we had measured the same person more than once at each time. Normally, we gather sufficient data to make this inference, but the RCI is used when we do not have data that allows us to estimate measurement error.

The RCI is not the only way to account for unestimated measurement error. Other methods for this general purpose include so-called "measurement error models," a diverse set of methods common in social sciences (Carroll \& Ruppert, 1996; Stefanski, 2000) that explicitly insert errors into other statistical models; structural equation modeling, a multivariate expansion of the general linear model capable of incorporating arbitrary latent errors; and meta-analysis, which augments study-level observations with sampling variance. The RCI can be seen as the simplest example of any of these methods. As such, when considering its use, an analyst should consider whether using the simplest available method of accounting 
for error is appropriate, or whether a more accurate method is available.

Because it is one among several options, disagreement over the RCI began almost immediately after its publication. Some revisions have been adopted (e.g., Christensen \& Mendoza, 1986), while others have yet to see wide uptake. Over the years, there have been numerous theoretical discussions of the RCI (e.g., Wise, 2004), clarifications of its appropriate uses (e.g., Langkaas et al., 2018), and suggested improvements to its formula. There is a diversity of opinion just in this last category: revised RCIs have included improvements to the estimation of the difference score (e.g., Hageman \& Arrindell, 1993, 1999); modifications of the estimate of error (e.g., Bruggemans et al., 1997), modifications to the estimates of standard deviation (e.g., Maassen, 2004), and generalizations (e.g., Hays et al., 2021). While many of these alternatives are clear improvements under certain conditions, none result in vastly superior estimates in every case (Atkins et al., 2005; Maassen, 2000; Ronk et al., 2016). Nevertheless, the 1991 RCI formula is definitively suboptimal in many circumstances (Duff, 2012; Maassen, 2004), and since it informs impactful decisions about individuals, even small improvements are important. As with any statistical test, preference among alternatives depends on the specific data and theory under consideration.

\section{When to use the RCI}

Since it is a relatively simple statistical test, determining when to use the RCI should be straightforward. Like all statistical tests, determining when to actually use the RCI can be challenging. This choice depends on the goals of the analysis, the available data, assumptions that can be justified, and available alternative solutions. Several criteria to consider before using the RCI are listed below and visualized as a suggested flow chart in Figure 1. If these criteria are not met, the RCI will be suboptimal, irrelevant, and/or harmful. For each criterion, alternatives are suggested and many others would be

possible as well. No alternative is ideal in every case, but the RCI addresses a problem of inadequate data, so the only universally preferable option to the RCI is to collect better data.

\section{Do you want to focus on difference scores?}

The RCI is a test of a difference score, and difference scores are not always the proper target of analysis. Difference scores only reflect treatment effects per se under limited circumstances (Kim \& 
Steiner, 2021; Langkaas et al., 2018). In many cases, difference scores can be misleading, particularly when compared across settings (Cuijpers et al., 2017). Difference scores are only even possibly relevant if basic measurement features are established: the scores exist on a continuous interval scale free of ceiling and floor effects. If two identical scale scores may come from two meaningfully different presentations (Fried \& Nesse, 2015), difference scores will be inconsistent. It may be rare to find PROMs of mental health symptoms that satisfy these measurement properties, but it is possible the assumptions are not devastating. Still, just using change scores may lead to avoidable problems.

If difference scores are not of interest, what might be? It has been argued that end-state functioning is more clinically relevant than pre-post change in medicine, and different tests of clinical significance might be appropriate if this is the case (Kendall et al., 1999). When these kinds of methods are applied along with change-score focused ones, they may yield different and even opposite findings (McAleavey et al., 2019). Additional methods that incorporate baseline severity, for instance, residualized gain scores and percent symptom reduction, are often preferrable, and enable other analyses. Using these alternatives obviates the RCI in many cases.

\section{Is any true score change, regardless of its magnitude, important?}

The RCI should not be used to indicate a meaningful change by itself, unless any true score change is important. The purpose of the RCI is to identify observed score changes that cannot plausibly be attributed to measurement error alone, meaning that they likely include some true score change, though this component could be infinitesimally small. Therefore, the RCI should be thought of as a way to tell if true change exists given certain assumptions, not that the true change matters.

The fact that the RCI aims to identify even very small true changes is why the RCI is sometimes called the "smallest detectable change" (SDC) or "minimum detectable change" (Hays \& Peipert, 2021). However, these terms are confusing, since the RCI is clearly not the smallest amount of true change that can be detected. The RCI is the largest plausible error in a difference score. Many true changes are not detected by the RCI, including true changes that are larger than the RCI. The RCI should only be considered the SDC if its definition of detection is the correct definition, a tautological loop. 
It is useful to distinguish here a very different construct from SDC, which might easily be confused for it: the "just-noticeable difference" (JND). Derived from signal detection theory, the JND is the smallest difference correctly detected a certain percent of the time, often $50 \%$. This criterion is often used to indicate how sensitive a system is to small changes. As an example, a standard ruler with millimeter ticks would have JND of $0.5 \mathrm{~mm}$. A real change the size of the JND will be detected $50 \%$ of the time, even if the person reading the ruler introduces random noise. As long as error is symmetric and has a mean of zero, the JND is independent of the measurement error. By extension, the JND for any psychometric instrument is half the smallest increment of that scale. Real changes at least that large will be detected at higher than chance accuracy. In contrast, the RCI is often an order of magnitude larger than the JND, making it very difficult to consider the RCI the "smallest detectable change." On the Patient Health Questionnaire-9 (PHQ-9; Kroenke et al., 2001), for example, the JND is 0.5 points, but a commonly-used RCI value is 6 points (Gyani et al., 2013), 12 times larger than the JND.

Because the RCI is only a test of 0 true score change, it should not be interpreted as a clinically meaningful change on its own. This is consistent with the use of the RCI as a "purely statistical" test (Jacobson et al., 1984; Jacobson \& Truax, 1991). Using the RCI as an indicator of how much change is important was explicitly not the intent of Jacobson \& Truax, who clearly recommended other tests be applied before concluding change is meaningful. Jacobson and colleagues write this about criteria that are based solely on the magnitude of a change score: "Without reference to some standard - such as normal functioning - these decision rules are likely to be subjective and arbitrary" (1984, p. 339).

Nevertheless, a large literature defining such benchmarks has emerged, especially with the Minimal Important Difference (MID; Turner et al., 2010). The MID is "the smallest difference in scores of a PRO measure that is perceived by patients as beneficial or harmful, and which would lead the clinician to consider a change in treatment" (Revicki et al., 2008, p. 103). In this context, the RCI is considered one of several "distribution-based" definitions of MID, while the major group of alternative methods are "anchor-based": meaningful changes identified through other methods (e.g., clinician evaluation) are associated with a corresponding amount of change on the measurement instrument. In 
MID literature, anchor-based methods are almost universally recommended over distributional approaches (Copay et al., 2008; Revicki et al., 2008; Turner et al., 2010; Wolpert et al., 2015).

Importantly, the RCI is a poor estimate, on many tests, of the MID. Ironically, the original proponents of the RCI were concerned that it would be overly sensitive (Jacobson et al., 1984, p. 344). This is strikingly opposed to empirical studies that have repeatedly shown the RCI to be insensitive compared to anchor-based MID estimates. For instance, Wolpert and colleagues (2015) reported that that the RCI had "low sensitivity" among several methods they investigated for determining clinically meaningful change. Turner and colleagues (2010) found that the RCI was consistently the largest value among options they tested. Copay and colleagues (2008) found the RCI (called MCD in their study) of moderate size, not minimal. Thissen and colleagues (2016) found the MID on the PROMIS pediatric measures to be around 2, while the RCI of those instruments in other studies (e.g., Pilkonis et al., 2014) is between 6-8 points. Cella et al. (2002) compared several methods for minimal change detection, finding that the SEM- the RCI divided by 2.77 - was close to the anchor-based method across a range of measures in their samples and recommend using these values. Overall, the RCI seems to be problematically inaccurate when interpreted as a measure of meaningful change.

Like Jacobson \& Truax, many authors use the RCI in conjunction with another test that they claim is more clinically meaningful. However, if a truly meaningful clinical test is available, the RCI may be obviated. We do not need to compute the RCI to know that a patient who previously had catatonic depression but now regularly completes tasks of daily living has changed: the RCI on a measure of depression is superfluous. Alternatively, how should we interpret a case in which there is no clinically meaningful change, but a reliable change is detected? Jacobson \& Truax (1991) called this case "Improved," but this seems like a very hollow definition of improvement if the change is not clinically meaningful. This is only plausibly "Improved" if there is some clinical value in the RCI's conclusion, which would make it unnecessary to combine a second clinical test.

Some have argued that when the RCI is larger than an estimate of MID (or differs from any other clinical test), the RCI should be used as the MID (e.g., Hays \& Peipert, 2021; Kemmler et al., 2010; King, 
2011). This contention is confusing, because the RCI and the MID are entirely different quantities (de Vet \& Terwee, 2010; Terwee et al., 2009). They can provide different levels of confidence and rely on entirely different data. Anchor-based MIDs group patients through empirical analysis of real or hypothetical changes, while the RCI groups patients through assumptions and estimates of stability. They are simply different values. There is no contradiction in finding that a change is likely to be clinically meaningful even if it is not as large as the RCI, nor is it a problem to say a particular change score is consistent with real change but is not large enough to be clinically meaningful.

If you believe only large true changes matter but wish to have conventional control over Type I errors, you can use several methods. Equivalence testing, for instance with two one-sided tests (Lakens et al., 2018) or a region of practical equivalence (Kruschke, 2018) can be useful, and the RCI can function as the error term in these models. Terwee and colleagues (Terwee et al., 2009) have demonstrated a similar method as well. These methods will generally be more conservative and less sensitive than the RCI, since they do not treat small true changes as meaningful. However, just like the RCI, none of these methods improve the data and therefore they do not solve the fundamental problem.

In sum, the RCI only indicates any non-zero true score change, so only means a change is important if any non-zero change is important. This may be a reasonable position, especially when considering negative changes (McAleavey, 2022a). But in many cases, people wish to make other claims about change, such as how likely a patient is to feel improved or how capable they are after treatment. The RCI has very limited value in such determinations and should not be relied on for those tasks.

\section{Are you only considering one patient (at a time)?}

The RCI is a test for an individual difference score, not a group. This means that you must want to know whether one person has changed or not. Comparing groups, for instance on the percentage of individuals achieving "reliable" change, is obviously possible (Blampied, 2017), but this group comparison is not directly tested by the RCI. Merely observing a higher frequency of large changes in one group does not indicate that the difference between groups is "real" or "meaningful," due to sampling variability that the RCI does not consider. In general, this type of responder analysis is vulnerable to 
nonnormally distributed outcomes and can be misleading (Snapinn \& Jiang, 2007).

Several methods exist to formally test the differences between groups in clinical significance. The simplest would be to test the percentages of those achieving RCI-level changes across groups using a chisquared test. Such tests are particularly vulnerable to non-normal distribution of change scores, however. Alternatively, you may be interested in whether the difference between groups' average outcomes is clinically important, and whether it is both clinically important and statistically reliable. If that is the case, other methods (De Boer et al., 2005; De Vet et al., 2001; 2009) may be more appropriate. The concept of clinical significance at the group level is fundamentally different from the RCI, so you must decide on what level of analysis you wish to make inferences.

Since we are interested in true score changes, and that the RCI is an estimate of measurement error, it may be useful to estimate the true score distribution from the observed scores (Trafimow, 2014). By computing the RCI we have already estimated the magnitude of measurement error, and therefore can subtract the error variance from the observed variance to recapture the true score distribution. While this method says nothing about individuals, it can provide an unattenuated estimate of the proportion of a given group improved and deteriorated. This method was used by McAleavey (2022a), finding that the true score distribution implied that $20 \%$ of individuals in evidence-based care and $36 \%$ of individuals in routine care settings deteriorated, as opposed to the RCI-based estimates of $6 \%$ and $13 \%$ respectively, which are attenuated by the RCI's insensitivity.

\section{Do population statistics apply to this specific person?}

Using an externally-derived $\mathbf{S}_{\text {diff }}$ implies that you have population estimates that sufficiently represent the individual in question. At minimum, the individual should be representative of the population on which the estimates are based. As the population statistics become less plausible replacements for individual scores, the accuracy of the $\mathrm{S}_{\text {diff }}$ and the RCI itself decreases.

There are at least two reasons why population estimates might not be useful substitutes for individual data, particularly on PROMs: individuals may differ from even a well-measured group average, and between-person variability is fundamentally different from within-person. The first reason is 
relatively simple. The measurement error considered by the RCI is a normally distributed variable with mean of 0 and an SD of $\mathrm{S}_{\text {diff. }}$ That is, it is unbiased, and we do not know exactly how large it is. However, some individuals likely have biased errors, meaning they consistently provide over- or under-estimated changes due to response styles, idiosyncratic interpretations, and the many secondary purposes that selfreport data can entail in treatment. Theoretically, this is the purpose of multiplying $\mathrm{S}_{\text {diff }}$ by 1.96: it allows for a larger than average error. However, this cannot account for biases. This also means that the RCI loses validity when either the individual or the population used for developing the RCI have floor or ceiling effects, since errors cannot have a mean of 0 at the bounds of scales (Maassen, 2000). Using a population-based estimate, therefore, means some individual will not be well-represented.

Second, the RCI is based on between-person estimates of variability, though we are interested in interpreting a within-person value. There are fundamental differences between the variability between persons and the variability within-person (Fisher et al., 2018). Some authors have therefore suggested the use of within-person estimates of variability in the RCI (e.g., Hays \& Peipert, 2021), though developing a truly person-specific variability estimate may require more than 10 observations per person. More advanced methods, such as item-response theory (Hays et al., 2021), can account for additional sources of variability in measurement error, and models that estimate person-specific measurement error without being purely idiographic (e.g., Williams et al., 2021) may become widely used in the future. In practice, taking this challenge seriously either entails not using the RCI or at minimum ensuring a very close match between population and individual, while also being even more cautious about interpretations as well.

\section{Do you have only one observed variable?}

If we have satisfied the above criteria, we are interested in estimating the measurement error in a single difference score using population statistics. This is difficult, inherently imprecise, and since it pertains to individual patients, should be considered high stakes. Therefore, we should be motivated to ensure we estimate measurement error as precisely as possible. As noted earlier, it is common to understand the RCI as a characteristic of a specific instrument, implying that error is only a function of the instrument. However, instruments are not the source of all measurement errors. Measurement errors 
are also caused by the people being measured and context of measurement, and several methods are available to separate their influence with sufficient data (e.g., Cernat \& Oberski, 2022; Morgan-Lopez et al., 2022). The RCI is only the optimal estimate if there are no other relevant observations.

In the analysis of groups of individuals, analysts routinely use covariates to statistically control for the effects of individual and contextual factors, which has the effect of altering our error estimates. There is no reason to ignore these variables when making a judgment about a specific individual, since we are already willing to incorporate data from external sources by using the RCI. A multiple-variable regression-based method has already shown improvements in accuracy over the RCI in at least one study (Temkin et al., 1999). Maassen (2000) notes that such regression methods have variable Type I error control depending on covariate levels, so such tools should be used with caution.

With that caution in mind, regression-based models could be extended to time-varying covariates as well. As an example, a patient whose PHQ-9 score increases by 7 points has reliably deteriorated on depression. A patient whose Work and Social Adjustment Scale (WSAS) score increases by 12 points has reliably improved functioning, based on the RCI (Amati, 2020). However, if one patient reports reliably deteriorated depression and reliably improved functioning, we should not treat these as unrelated. Such discrepant scores are unlikely. A more clinically and statistically robust interpretation is that there is greater error (equivalently, idiosyncrasy) in these scores than expected. In this case $\mathrm{S}_{\text {diff }}$ may be an underestimate of error, so we should not be confident in the independent RCIs. Of course, the patient could be experiencing this combination, but this mismatch should again tell us that more data is needed.

Since the RCI is an assumption-heavy substitute for measurement error, we should want to make this substitution with care and consideration. When there is more data than a single variable available, that means developing a more complex model and considering all observations.

\section{Do you have only two observations of that variable?}

The limitation of the RCI to two-timepoint difference scores was not elaborated on in the 1984 and 1991 papers, perhaps because the RCI was devised at a time when pre-post outcomes were typical study design. However, it is increasingly common that more than two measurements are collected during 
treatment, often with 4-20 observations per person. The RCI cannot accommodate this. This leads to researchers discarding these interim observations when considering reliable change, resulting in research waste. In Jacobson et al. (1984), the authors state that a proper single-subject designed study would "obviate the need for determining that the change is statistically reliable" (1984, p. 345). Other authors have noted this limitation of the RCI and recommended the field use more time points (Speer, 1999). If you are planning to assess change, collecting more than two timepoints seems like a good idea; if you have more than two observations, use all available data. However, with more than two observations per person, the number of analytic options grows dramatically. Analysts must justify their operationalizations of reliable change: Some options may be acceptable in one case and inadmissible in another.

As we have seen, with only two observations, we have no ability to estimate measurement error. With three or more observations, person-specific error can be estimated, though it may take many observations before estimates are accurate (Borckardt et al., 2008; Estabrook et al., 2012). In lieu of that, we could compute separate linear regressions for each person, using the resulting $p$-value for the effect of time on the outcome as an indicator of the reliability of a trend. This has appeal but does not explicitly account for measurement error, the resulting errors will generally be overestimates of measurement error, since they will include true fluctuations as well. Longitudinal hierarchical modeling is another option (Bauer et al., 2004). In common applications it does not account for measurement error, though because individuals' trajectories will be systematically shrunk towards the group mean to a greater extent if their scores are highly variable, extreme, or there are not many observations per person, the noisiest data will be treated as error more often. Unsurprisingly, previous applications have not found high convergence with other methods of reliable change determination (Bauer et al., 2004; Speer \& Greenbaum, 2002).

If the basic assumptions of the RCI are acceptable, using more than two observations will reduce uncertainty, often with surprising efficiency, even with substantial measurement error (Donaldson, 2008). A general multiple-timepoint extension of the RCI, linear regression with measurement error, is already implemented in the open-source software R (McAleavey, 2022b).

Many methods including those just discussed could be justified in certain applications with more 
than two data points. While no specific alternative will be advisable in every case, if more than two data points are available and relevant to the analysis, the RCI is wasteful and inaccurate. There is limited justification to prefer an inadequate test that is simple over a superior test that requires effort.

\section{Do you want to categorize a continuous variable?}

The RCI is often used to separate continuous outcomes into categories. There are many reasons why discretizing continuous variables is frequently admonished by statisticians (e.g., Altman \& Royston, 2006). The most basic is that a continuous variable, even one with measurement error, has more information than a derivative categorical variable. Outcomes of mental healthcare, especially as measured in PROMs, are often continuously (or nearly-continuously) distributed. Categorization is a particular choice that requires justification since it will always result in lost information.

The use of categories also leads to a common misinterpretation of scores that do not meet the reliable change threshold. Many analysts treat these scores as "unchanged" or reflecting "no change" (Langkaas et al., 2018). This started with the 1984 paper, where the authors write "It is nonsensical to speak of clinically significant treatment effects when no change has occurred" (Jacobson et al., 1984, p. 343). This is an instance of the common error of assuming that absence of evidence is evidence of absence (Altman \& Bland, 1995; Greenland et al., 2016). Because the RCI is a NHST, failing to reject the null hypothesis does not provide evidence that the patient is unchanged: we just do not have sufficient evidence. Unless the difference score is 0 , some change has been observed. A difference score larger than the RCI is only probably "changed," and there is no justification for treating scores below it as "unchanged," yet this nomenclature is common (Donaldson, 2008; Wise, 2004).

Even though it is generally not a good idea to categorize continuous variables, and RCI categories are frequently misinterpreted, there are times when categorization is desirable. Applied decisions are often categorical, for instance: Should a patient receive additional treatment or not? At some point, categorization must occur to answer this question, but this does not mean that the RCI's categorization is the proper one. Instead, decision-related categorization should reflect estimates of something relevant to the decision. Depending on the specific scenario, a decision to offer additional treatment may be justified 
by a determination that the patient has already improved and will continue to do so, has experienced a worsening of symptoms, has consistently attended sessions, has experienced gains that are highly unlikely to be due to chance alone, or any number of other considerations. The RCI is only relevant to a very small portion of this decision. Because of this, simplifying the change score to an RCI category limits the inputs to an important decision. This is especially important for cases near the cut point, who may be assigned very different categories for very similar outcomes: an individual whose difference score suggests a $4 \%$ chance of no change might be categorized as reliably changed, while a near-identical change with $6 \%$ likelihood would be categorized as not. Treating such similar individuals so differently does not "account for measurement error" in any meaningful sense, it introduces new errors that might go unnoticed.

Instead, it might be better to rely on continuous markers. A continuous approach closely related to the RCI is to compute the implied likelihood of true change greater than 0 for an individual's observed difference score, assuming the same magnitude of measurement error that the RCI uses. This is not complex if we have already estimated $\mathrm{S}_{\text {diff, }}$ in fact it is simple, interpretable, and familiar in statistical and clinical practice. For instance, the question, "How likely is it that Mac grew during the month?" could be operationalized as asking what percent of a Gaussian curve centered at the observed difference with $\mathrm{SD}=$ $\mathrm{S}_{\text {diff }}$ is above 0 . The answer is $92 \%$. That is, even based on our noisy instrument, there seems to be a $92 \%$ chance that Mac grew during the month. This is the same information summarized earlier by the RCI, which concluded that we were not confident Mac grew. While both conclusions stem from the same assumptions and data, they seem to conflict. Various continuous alternatives could easily be derived, such as the original RC index (Eq. 1). Again, no single solution is optimal in every case, except collecting more data. If categorization is desirable, the RCI is not necessarily the correct tool.

\section{Do you intend to conduct any additional analyses after categorization?}

Even if categories are desirable, they should not be used if additional analyses will be conducted. Because the RCI discards information through categorization, it is a mistake to use this newly-categorical variable as a predictor of other outcomes or as an outcome in a separate model. The underlying continuous variable will be superior on average, and in some cases vastly superior, to using categories 
(Altman \& Royston, 2006). As predictors, categorical variables have limited precision and increased noise compared to continuous underlying variables (Royston et al., 2006). Individuals in a "reliably changed" group may have outcomes that range from medium to extremely large relative to error, while those in the "unclassified" group may have moderate differences in opposite directions from each other.

The use of RCI-based categories as a predictor occurs in research in several ways. For instance, short-term treatments may have longer-term effects, and in those cases, the outcome of treatment might be used as a predictor of the longer-term effect. In this case, it may seem reasonable to separate individuals into groups based on their RCI and look for differential long-term impact between those groups. Such a responder analysis is potentially a mistake (Snapinn \& Jiang, 2007). Instead, if treatment outcome is to be an interim step of analysis, that outcome should be continuous if possible.

Psychometrically based precision mental healthcare entails many assessments and prediction models, for which using a categorization-first approach is costly. If a predictive model's dependent variable is categories of change, that predictive model has unnecessary noise in its computation. If that categorical model is then applied to another individual, it redoubles noise, like making a photocopy of a photocopy. This process inserts uncertainty using the RCI (as assumed measurement error), inserts more via categorization, and then adds even more uncertainty as prediction error. We could improve this by developing models for continuous outcomes, predicting a range of likely outcomes for an individual that incorporates measurement and prediction error, and then estimating the likelihood of non-zero change.

\section{Is there any cost to false negatives?}

An important benefit of the RCI is that it controls the Type I error rate, which are false-positive detections of change. In NHST for scientific studies, the Type I error rate is controlled at a conventional level (often .05), because these errors are particularly serious in the context of falsification-based science. However, the rationale for applying this convention to individual change scores is not clear. If false positive errors are the sole concern, the RCI may be appropriate. However, preventing Type I errors often increases the likelihood of Type II errors, which occur when we fail to detect a true effect. In scientific studies, researchers can decrease the chance of false negative inference in many ways, including 
increasing the number of people in a sample or changing the measurement strategy. The RCI fails to consider Type II errors at all. Therefore, if Type II errors have any cost (e.g., an ineffective or harmful treatment may be continued when true deterioration is undetected), the RCI is probably unacceptable.

Taking Type II errors into account, it is informative to consider the RCI's total accuracy, plotted as a function of true change in Figure 2. As can be seen, the RCI is incorrect most of the time for true changes smaller than the RCI itself. For true changes greater than the RCI, the RCI still leads to incorrect conclusions quite often. The RCI is only $95 \%$ correct if the true change is exactly 0 (which is the only case in which Type I error rates pertain), or if the true change is greater than 3.6 times $\mathrm{S}_{\text {diff. }}$ Such changes are generally obvious without statistics. Ferrer and Pardo (2019) report false-negative rates from $32 \%$ to 99.9\%, including situations with extremely large effects. Most small-to-moderate true changes will not be correctly detected. Since we have already established that we are interested in even very small true changes, the RCI's inaccuracy with such small changes is a serious issue. This may result in providers dismissing true negative experiences of patients as not "real."

To put this on the scale of a commonly used PROM, the 20-item PCL-5 has an RCI value of 18 scale points (Marx et al., 2021). This means that there is only a $50 \%$ chance that a patient with a true change of 18 points has a "reliable" change, and that individuals can only be $95 \%$ accurately categorized with the RCI if their true change scores are greater than 33 scale points or exactly 0 . Given that pre-post differences in highly effective PTSD treatments are routinely less than 30 points on this measure (e.g., Bækkelund et al., 2022), the RCI is $95 \%$ accurate only rarely.

Gelman and Carlin (2014) suggest that researchers should also consider Type S (how likely is an estimate to have the incorrect sign) and Type $\mathrm{M}$ errors (when an effect is significant, how much larger is its estimate than the true value). Applying this to the RCI shows that reliable changes may be substantial overestimates of smaller true changes. For instance, a true difference score equal to $\mathrm{S}_{\text {diff }}$ should generate an observed difference score greater than the RCI $17 \%$ of the time: that is, the RCI has $83 \%$ Type II error in this case. But when the RCI is correct, the observed difference score will be 2.5 times greater than the true difference score, on average (see the Supplement for detail). This is a huge overestimate and is 
therefore a highly unreliable difference, in a meaningful sense. To be clear, these problems are not specific to the RCI, they are due to applying cutpoints to error-prone continuous data and $p$-value conventions. Still, it demonstrates that RCI-based categories should be treated with caution, and that difference scores greater than and less than the RCI value contain potentially large measurement errors.

Several authors have argued that the RCI is too conservative for clinical use, and advocated for less extreme values as the multiple of $\mathrm{S}_{\text {diff }}$ in Eq. 2. Wise (2004) advocated for using 1.96, 1.28, and 0.84 to indicate different confidence levels, Donaldson (2008) advocated for 1.96 and 0.60, and recently, Peipert and colleagues (2022) recommend 0.994 and 0.674 . While any of these options are possible, if a cutpoint is desired, the least arbitrary is simply 0 . That is, we could treat any positive observed difference as if it represents positive true score change, and the same with any negative observed differences. Because this will produce incorrect inferences relatively often when the observed difference scores are small, we might expect such a method to be inaccurate. However, it is surprisingly accurate, as can be seen in Figure 2. In contrast to the RCI, we can see that its accuracy is always over $50 \%$, and the range of $95 \%$ accuracy is considerably larger than the RCI.

Moreover, the RCI will tend to identify people with unusually large measurement errors as reliably changed, while failing to detect many accurately-measured smaller changes. This leads to bias. In the scientific literature, this is known as the statistical significance filter and results in over-confidence in certain results while dismissing others (Vasishth et al., 2018). By extension, if we interpret difference scores over the RCI as reliable, we know that they will be over-estimates of true changes, and are not, therefore, reliable. In contrast, the sign of the difference score tends to be most accurate for scores with small measurement error components, which is desirable: the most accurately measured observations will be most accurately interpreted. When true changes are small to moderate, the RCI tends to identify the least accurately measured scores as "reliably" changed, and many of the most accurately measured ones as not. These discrepancies are less drastic with higher SNR, but in every case, even when the RCI is correct it will tend to be biased. This is shown in Figure 3 and details can be found in the Supplement. While there may be reasons to be conservative in decision-making, if unbiased and/or accurate 
evaluations are important, any non-zero cutpoint is suboptimal. Therefore, the RCI should not be used when decision accuracy per se is valuable, nor when there are any costs associated with Type II errors, but rather when false positives are the only costly outcome. However, if any costly outcomes might occur at all, using just two observations from a noisy instrument is misguided. Again, collect better data.

\section{Do you have an appropriate reliability estimate?}

The reliability estimate is the most important variable in the RCI formula, yet it has been surprisingly flexibly interpreted. Only reliability estimates from repeated administrations (e.g., test-retest reliability) are appropriate for the RCI, because they estimate how dependable a score is with repeated administrations (Guttman, 1945). Test-retest reliability was the only form of reliability suggested by Jacobson and Truax (1991) and Jacobson, Follette, and Revenstorf (1984), and it is clearly appropriate. Similar multiple-timepoint reliability assessments (e.g., within-person ICC) are theoretically similar and may be justifiable as well. Reliability indicators based on a single assessment are neither equivalent nor interchangeable with test-retest (American Educational Research Association et al., 2014; Cattell, 1982; McCrae et al., 2011; Sijtsma, 2009; Viswanathan, 2005). These are different aspects of reliability: "Different reliability ... coefficients may appear to be interchangeable, but the different coefficients convey different information" (American Educational Research Association et al., 2014, p. 38).

The use of Cronbach's alpha in the RCI, which has become common (e.g., Bauer et al., 2004; Beckstead et al., 2003; Greeke et al., 2017; Martinovich et al., 1996; Pilkonis et al., 2014; Youngstrom et al., 2017) is particularly problematic. Coefficient alpha has been criticized for being unrealistic (Flora, 2020), overly sensitive to the length of a test (Cronbach, 1951), and for usually being among the lowest estimates of internal consistency reliability (Revelle \& Condon, 2019; Sijtsma, 2009). Several authors have advocated against alpha in several applications (e.g., Flake et al., 2017; Sijtsma, 2009), including in the RCI (e.g., Ferrer \& Pardo, 2014; McCrae et al., 2011). For these reasons, we should expect using alpha in the RCI will lead to longer instruments having smaller RCIs than shorter instruments even when they are not more precise measures of change, and that alpha-based RCIs will be insensitive to change.

Alpha and test-retest differ systematically. McCrae and colleagues (2011) note that while many of 
the sources of error that are detected by internal consistency are also detected by test-retest reliability (e.g., respondent error, item ambiguity), some sources of error are detected by internal consistency alone (e.g., irrelevant items, heterogeneous items), while others are only measured by test-retest (true state variability). Test-retest treats idiosyncratic-but-stable interpretations and between-person variability in the relative value of item content as true score variance, while internal consistency treats these as noise. In cases where individuals may idiosyncratically-but-consistently interpret items, the difference between internal consistency and test-retest can be large. This suggests that the two criteria could be different and not directly linked. An alternative conception is simulated by Ferrer and Pardo (2014), who assume testretest reliability subsumes internal consistency. In their case, alpha led to a high false positive rate in the RCI. Test-retest and internal consistency are also empirically differentiable: the correlation between them across measures of personality is only $r=.25$ (Chmielewski \& Watson, 2009), and test-retest was more closely related to validity than internal consistency (McCrae et al., 2011).

Further, the interval of test-retest is essential to generate a valid reliability estimate (Cattell, 1966, 1982; Chmielewski \& Watson, 2009; Watson, 2004). Regarding this, the AERA/APA/NECM Standards of Psychological Test Development (2014) state: “The test is administered and then, after a brief period during which the examinee's standing on the variable being measured would not be expected to change, the test (or a distinct but equivalent form of the test) is administered a second time" (p. 34). The appropriate interval depends on the variable: "For a state variable ... where fairly rapid changes are common, scores generated on two successive days would not be considered replications" (p. 35). If true scores on the variable could change during an interval, that interval is longer than a test-retest interval.

This means that psychological assessments must have retest intervals shorter than the interval with which they will be applied. It is common to see 1-week, 2-week, and even 4-week (e.g., Beard et al., 2016) intervals used to determine the RCI. Using a measure weekly (e.g., at psychotherapy sessions) implies the underlying construct can change at least that quickly, invalidating these retest intervals. Many common targets of psychological interest change daily or more frequently (Fisher et al., 2017, 2018; Molenaar, 2004). Indeed, this a core premise of some recent models of psychopathology (e.g., Robinaugh 
et al., 2020). If there is reason to expect that true score change may occur over one week, a test-retest period of less than a week is needed. If change occurs daily, intervals shorter than a day are required.

There are some costs to shorter retest intervals in psychological testing. For one, near-immediate retest intervals for self-report data risk introducing new errors through memory effects (when the participant seeks to be consistent across administrations) and frustration/fatigue effects (when repetitive questioning decreases care and attention). Memory effects can be mitigated with brief intervals between test and retest, and/or the use of interim distraction tasks. Frustration and inattention can be combated with clear and ethical description of research procedures, and pre-registered removal of participants who provide hasty responses. The optimal interval must balance the chance of true score changes during the interval with these induced errors, so a few minutes or hours may be ideal.

Note that the interval between our observations of interest (the one-month period between Mac's measurements) does not dictate the proper interval for test-retest, except that the test-retest interval must be the shorter of the two. It is appropriate to use an immediate test-retest interval in our height example, even though we wish to infer something about Mac's height over the course of a month, and indeed this is the only appropriate interval to avoid real variations in height. Reliability is part of the RCI to estimate "the fluctuations of an imprecise measuring instrument," not the variability in true scores. Including any true variation in the test-retest coefficient will underestimate reliability (Chmielewski \& Watson, 2009).

It might be noted that many PROMs are retrospective, asking examinees to rate items over a previous period such as 1 or 2 weeks. This does not change the need for short test-retest intervals. The accuracy of retrospective evaluation as a facsimile of experience is questionable, since it is not clear that responses truly reflect the period in question. However, if participants were able to provide accurate responses, the timeframe of retrospective review provides a false sense of stability of scores. A scale that asks participants to rate their symptoms over the past seven days only covers $86 \%$ of the same assessment period on two successive days, $71 \%$ if repeated 48 hours apart, and by 1 week, there is no shared assessment period whatsoever between two administrations. Even a perfect 1-week retrospective instrument with no error would achieve accurate test-retest estimation over a 1-week interval only if every 
participant's true score remained stable, relative to all other participants, for 2 full weeks. On some constructs this may be a reasonable assumption, and on others it is not.

Generally, we should expect shorter test-retest intervals to generate higher reliability estimates and more sensitive RCIs than currently used. However, same-day test-retest correlations will not be exactly 1. For example, Wallis (2013) administered the CES-D (Radloff, 1977) twice successively. The CES-D total score had an immediate test-retest correlation of $r=.96$, considerably higher than the Cronbach's alpha reported in the same sample $(\alpha=.88)$ as well as the alphas $(\alpha \mathrm{s}=[.84, .90])$ and correlations across 2 weeks to 12 months $(r s=[.32, .67]$; Radloff, 1977). Similarly, the Hamilton Rating Scale for Depression had one-day test-retest values of $r=.96$ and .98 but lower values for longer intervals (Trajković et al., 2011). A recent example can be found in Dejonckheere et al. (2022). Those authors found notable instability on single item measures assessing momentary affect, which should be highly unstable over time. This shows that some assessments may not be very dependable at this scale.

Small differences in reliability coefficients may not seem substantial. We may wonder whether the use of $\alpha=.88$ rather than $r=.96$ really matter. This does matter, for two reasons. First, this change is a dramatic improvement in signal detection, and second because each of these is merely an estimate with its own uncertainty. We can see this by computing relative RCI values. Keeping the estimate of $s_{1}$ constant, a change from $r_{x x}=.88$ to $r_{x x}=.96$ would reduce the size of the RCI by $43 \%$. That will have the effect of making the RCI identify many more "reliable" changes. This, combined with failing to differentiate between different types of reliability estimates, encourages researchers to use the highest available estimate of reliability, regardless of its source or rationale. However, if more than one estimate is available, the highest one is unlikely to be the most accurate. It may be better to rely on averages of many separate appropriate test-retest reliability estimates for the RCI.

\section{Is there any potential practice effect?}

In some cases, test scores change on a second administration merely because the test-taker has experience with the test itself, not because their true score changes. This is a practice effect and is especially important in neuropsychology. Some tests have substantial practice effects, while practice 
effects are small on others. While they may never be truly zero, applied researchers often assume no practice effect is present, and use the RCI. If there is concern that practice effects are present, alternative formulae are available (Chelune et al., 1993; Duff, 2012; Maassen et al., 2009). One essential modification of the RCI formula is that these alternatives do not consider the raw difference score, but rather the difference compared to the average difference in a control sample. Given the simplicity of this adjustment, one of these examples should almost always be preferred if practice effects might occur.

\section{Is the typical spread of scores dependent on time?}

In hindsight, it seems rather vague that Jacobson \& Truax (1991) provide only this for the definition of $s_{1}$ : the "standard deviation of the control group, normal population, and pretreatment experimental group" ( $\mathrm{p} 14)$. These will be meaningfully different in many circumstances, including if the control group is not the normal population; the normal population distribution is heavily affected by floor effects; the pretreatment experimental group has a restricted range; or any of the samples were collected in a meaningfully different context than the treatment sample. Additionally, the SD may change as a function of time even if the average difference score is 0 . Luckily, superior forms of the RCI that account for this are available and easy to calculate. Readers are directed to the works of Maassen (2004; Maassen et al., 2009), who provides alternative formulae which should almost always be preferred to the RCI.

\section{Use the RCI?}

Otherwise, the RCI as recommended by Jacobson \& Truax (1991) may be appropriate.

\section{Discussion}

Despite its popularity, the circumstances in which the RCI will be helpful are paradoxically limited. You must be interested in analyzing a changing process, yet only have two observations. You must want excellent control over false positive, but no control of false negative errors. You must take measurement error to be a serious concern, but generalization across individuals to be no challenge at all. You must be interested in categorizing individuals, but willing to fail to categorize a large portion of them. You must believe any small true change is important, but use a method that only accurately detects large true changes. It is hard to avoid concluding that the RCI is less accurate than desired. In clinical 
application, the RCI likely adversely affects individuals, especially by failing to detect real changes. This calls into question the utility of the RCI, particularly in the evaluation of psychological symptoms.

It could still be the case that the RCI is acceptable in some circumstances. Most of the problems identified in this manuscript tend to make the RCI less sensitive than it should be, so it will be more appropriate when that is acceptable. And of course, the RCI can be justified for some purposes, such as a comparison to past research or as a temporary approximation. Nevertheless, better methods should be preferred, and such practicalities do not justify its use without clear acknowledgment of the limitations. The RCI attempts to answer to an important, but fundamentally unanswerable, question: How confident can we be in the difference between two scores from one individual, given that we believe those scores have measurement error? The RCI can be reasonable if its assumptions are met, but it cannot overcome the fundamental problem of insufficient data. Collecting better data is the only real solution. 


\section{Disclosures}

Author Contributions: Conceptualization, Methodology, Validation, Formal Analysis, Writing Original Draft, Writing - Review \& Editing, Visualization: A. McAleavey

Conflicts of Interest: The author(s) declare that there were no conflicts of interest with respect to the authorship or the publication of this article.

Preprints: A previous version of this manuscript has been available publicly since November 22, 2021 at https://osf.io/3kthg/. The current version and its supplement have been made available here: 


\section{References}

Altman, D. G., \& Bland, J. M. (1995). Statistics notes: Absence of evidence is not evidence of absence. BMJ, 311(7003), 485. https://doi.org/10.1136/bmj.311.7003.485

Altman, D. G., \& Royston, P. (2006). The cost of dichotomising continuous variables. BMJ (Clinical Research Ed.), 332(7549), 1080-1080. PubMed. https://doi.org/10.1136/bmj.332.7549.1080

Amati, F. (2020). Ethnicity, social function and psychological treatment outcomes in patients with common mental disorders: A large retrospective naturalistic cohort analysis.

American Educational Research Association, American Psychological Association, \& National Council on Measurement in Education. (2014). Standards for educational and psychological testing. American Educational Research Association.

American Psychological Association. (2021, November 16). Most Cited Articles. https://psycnet.apa.org/PsycARTICLES/journal/ccp/mostcited

Atkins, D. C., Bedics, J. D., McGlinchey, J. B., \& Beauchaine, T. P. (2005). Assessing clinical significance: Does it matter which method we use? Journal of Consulting and Clinical Psychology, 73(5), 982.

Bækkelund, H., Endsjø, M., Peters, N., Babaii, A., \& Egeland, K. (2022). Implementation of evidencebased treatment for PTSD in Norway: Clinical outcomes and impact of probable complex PTSD. European Journal of Psychotraumatology, 13(2), 2116827.

Bauer, S., Lambert, M. J., \& Nielsen, S. L. (2004). Clinical significance methods: A comparison of statistical techniques. Journal of Personality Assessment, 82(1), 60-70.

Beard, C., Hsu, K., Rifkin, L., Busch, A., \& Björgvinsson, T. (2016). Validation of the PHQ-9 in a psychiatric sample. Journal of Affective Disorders, 193, 267-273.

Beckstead, D. J., Hatch, A. L., Lambert, M. J., Eggett, D. L., Goates, M. K., \& Vermeersch, D. A. (2003). Clinical significance of the Outcome Questionnaire (OQ-45.2). The Behavior Analyst Today, $4(1), 86$.

Blampied, N. M. (2017). Analyzing therapeutic change using modified Brinley plots: History, 
construction, and interpretation. Behavior Therapy, 48(1), 115-127.

Blampied, N. M. (2022). Reliable change and the reliable change index: Still useful after all these years? The Cognitive Behaviour Therapist, 15, e50.

Bland, J. M., \& Altman, D. G. (1996). Measurement error. BMJ: British Medical Journal, 312(7047), 1654.

Borckardt, J. J., Nash, M. R., Murphy, M. D., Moore, M., Shaw, D., \& O’Neil, P. (2008). Clinical practice as natural laboratory for psychotherapy research: A guide to case-based time-series analysis. American Psychologist, 63(2), 77.

Bruggemans, E. F., Vande Vijver, F. J., \& Huysmans, H. A. (1997). Assessment of cognitive deterioration in individual patients following cardiac surgery: Correcting for measurement error and practice effects. Journal of Clinical and Experimental Neuropsychology, 19(4), 543-559.

Carroll, R. J., \& Ruppert, D. (1996). The Use and Misuse of Orthogonal Regression in Linear Errors-inVariables Models. The American Statistician, 50(1), 1-6.

Cattell, R. B. (1966). Patterns of change: Measurement in relation to state dimension, trait change, lability, and process concepts. In Handbook of multivariate experimental psychology (pp. 355402). Rand McNally.

Cattell, R. B. (1982). The clinical use of difference scores: Some psychometric problems. Multivariate Experimental Clinical Research, 6(2), 87-98.

Cella, D., Eton, D. T., Lai, J.-S., Peterman, A. H., \& Merkel, D. E. (2002). Combining anchor and distribution-based methods to derive minimal clinically important differences on the Functional Assessment of Cancer Therapy (FACT) anemia and fatigue scales. Journal of Pain and Symptom Management, 24(6), 547-561.

Cernat, A., \& Oberski, D. (2022). Estimating Measurement Error in Longitudinal Data Using the Longitudinal MultiTrait MultiError Approach. Structural Equation Modeling: A Multidisciplinary Journal, O(0), 1-12. https://doi.org/10.1080/10705511.2022.2145961

Chelune, G. J., Naugle, R. I., Lüders, H., Sedlak, J., \& Awad, I. A. (1993). Individual change after 
epilepsy surgery: Practice effects and base-rate information. Neuropsychology, 7(1), 41.

Chmielewski, M., \& Watson, D. (2009). What is being assessed and why it matters: The impact of transient error on trait research. Journal of Personality and Social Psychology, 97(1), 186.

Christensen, L., \& Mendoza, J. L. (1986). A method of assessing change in a single subject: An alteration of the RC index. Behav Ther, 17, 305-308.

Copay, A. G., Glassman, S. D., Subach, B. R., Berven, S., Schuler, T. C., \& Carreon, L. Y. (2008). Minimum clinically important difference in lumbar spine surgery patients: A choice of methods using the Oswestry Disability Index, Medical Outcomes Study questionnaire Short Form 36, and pain scales. The Spine Journal, 8(6), 968-974.

Cronbach, L. J. (1951). Coefficient alpha and the internal structure of tests. Psychometrika, 16(3), 297334.

Cuijpers, P., Weitz, E., Cristea, I., \& Twisk, J. (2017). Pre-post effect sizes should be avoided in metaanalyses. Epidemiology and Psychiatric Sciences, 26(4), 364-368.

De Boer, M. R., De Vet, H. C., Terwee, C. B., Moll, A. C., Völker-Dieben, H. J., \& Van Rens, G. H. (2005). Changes to the subscales of two vision-related quality of life questionnaires are proposed. Journal of Clinical Epidemiology, 58(12), 1260-1268.

De Vet, H. C., Bouter, L. M., Bezemer, P. D., \& Beurskens, A. J. (2001). Reproducibility and responsiveness of evaluative outcome measures: Theoretical considerations illustrated by an empirical example. International Journal of Technology Assessment in Health Care, 17(4), 479487.

de Vet, H. C., \& Terwee, C. B. (2010). The minimal detectable change should not replace the minimal important difference. Journal of Clinical Epidemiology, 63(7), 804-805.

Dejonckheere, E., Demeyer, F., Geusens, B., Piot, M., Tuerlinckx, F., Verdonck, S., \& Mestdagh, M. (2022). Assessing the reliability of single-item momentary affective measurements in experience sampling. Psychological Assessment.

Donaldson, G. (2008). Patient-reported outcomes and the mandate of measurement. Quality of Life 
Research, 17(10), 1303-1313.

Duff, K. (2012). Evidence-based indicators of neuropsychological change in the individual patient: Relevant concepts and methods. Archives of Clinical Neuropsychology, 27(3), 248-261.

Estabrook, R., Grimm, K. J., \& Bowles, R. P. (2012). A Monte Carlo simulation study of the reliability of intraindividual variability. Psychology and Aging, 27(3), 560.

Ferrer, R., \& Pardo, A. (2014). Clinically meaningful change: False positives in the estimation of individual change. Psychological Assessment, 26(2), 370.

Ferrer, R., \& Pardo, A. (2019). Clinically meaningful change: False negatives in the estimation of individual change. Methodology: European Journal of Research Methods for the Behavioral and Social Sciences, 15(3), 97.

Fisher, A. J., Medaglia, J. D., \& Jeronimus, B. F. (2018). Lack of group-to-individual generalizability is a threat to human subjects research. Proceedings of the National Academy of Sciences, 115(27), E6106-E6115.

Fisher, A. J., Reeves, J. W., Lawyer, G., Medaglia, J. D., \& Rubel, J. A. (2017). Exploring the idiographic dynamics of mood and anxiety via network analysis. Journal of Abnormal Psychology, 126(8), 1044-1056. https://doi.org/10.1037/abn0000311

Flake, J. K., Pek, J., \& Hehman, E. (2017). Construct validation in social and personality research: Current practice and recommendations. Social Psychological and Personality Science, 8(4), 370 378.

Flora, D. B. (2020). Your coefficient alpha is probably wrong, but which coefficient omega is right? A tutorial on using R to obtain better reliability estimates. Advances in Methods and Practices in Psychological Science, 3(4), 484-501.

Fried, E. I., \& Nesse, R. M. (2015). Depression sum-scores don't add up: Why analyzing specific depression symptoms is essential. BMC Medicine, 13(1), 1-11.

Gelman, A., \& Carlin, J. (2014). Beyond power calculations: Assessing type S (sign) and type M (magnitude) errors. Perspectives on Psychological Science, 9(6), 641-651. 
Greeke, E. E., Chua, A. S., Healy, B. C., Rintell, D. J., Chitnis, T., \& Glanz, B. I. (2017). Depression and fatigue in patients with multiple sclerosis. Journal of the Neurological Sciences, 380, 236-241.

Greenland, S., Senn, S. J., Rothman, K. J., Carlin, J. B., Poole, C., Goodman, S. N., \& Altman, D. G. (2016). Statistical tests, P values, confidence intervals, and power: A guide to misinterpretations. European Journal of Epidemiology, 31(4), 337-350. https://doi.org/10.1007/s10654-016-0149-3

Guttman, L. (1945). A basis for analyzing test-retest reliability. Psychometrika, 10(4), 255-282.

Gyani, A., Shafran, R., Layard, R., \& Clark, D. M. (2013). Enhancing recovery rates: Lessons from year one of IAPT. Behaviour Research and Therapy, 51(9), 597-606.

Hageman, W. J., \& Arrindell, W. A. (1993). A further refinement of the reliable change (RC) index by improving the pre-post difference score: Introducing RCID. Behaviour Research and Therapy, $31(7), 693-700$.

Hageman, W. J., \& Arrindell, W. A. (1999). Establishing clinically significant change: Increment of precision and the distinction between individual and group level of analysis. Behaviour Research and Therapy.

Hays, R. D., \& Peipert, J. D. (2021). Between-group minimally important change versus individual treatment responders. Quality of Life Research, 1-8.

Hays, R. D., Spritzer, K. L., \& Reise, S. P. (2021). Using Item Response Theory to Identify Responders to Treatment: Examples with the Patient-Reported Outcomes Measurement Information System (PROMIS®) Physical Function Scale and Emotional Distress Composite. Psychometrika, 1-12.

Jacobson, N. S., Follette, W. C., \& Revenstorf, D. (1984). Psychotherapy outcome research: Methods for reporting variability and evaluating clinical significance. Behavior Therapy, 15(4), 336-352.

Jacobson, N. S., Roberts, L. J., Berns, S. B., \& McGlinchey, J. B. (1999). Methods for defining and determining the clinical significance of treatment effects: Description, application, and alternatives. Journal of Consulting and Clinical Psychology, 67(3), 300.

Jacobson, N. S., \& Truax, P. (1991). Clinical Significance: A Statistical Approach to Defining Meaningful Change in Psychotherapy Research. Journal of Consulting and Clinical Psychology, 
$59(1), 12-19$.

Kemmler, G., Zabernigg, A., Gattringer, K., Rumpold, G., Giesinger, J., Sperner-Unterweger, B., \& Holzner, B. (2010). A new approach to combining clinical relevance and statistical significance for evaluation of quality of life changes in the individual patient. Journal of Clinical Epidemiology, 63(2), 171-179.

Kendall, P. C., Marrs-Garcia, A., Nath, S. R., \& Sheldrick, R. C. (1999). Normative comparisons for the evaluation of clinical significance. Journal of Consulting and Clinical Psychology, 67(3), 285.

Kim, Y., \& Steiner, P. M. (2021). Gain scores revisited: A graphical models perspective. Sociological Methods \& Research, 50(3), 1353-1375.

King, M. T. (2011). A point of minimal important difference (MID): A critique of terminology and methods. Expert Review of Pharmacoeconomics \& Outcomes Research, 11(2), 171-184.

Kroenke, K., Spitzer, R. L., \& Williams, J. B. (2001). The PHQ-9: Validity of a brief depression severity measure. Journal of General Internal Medicine, 16(9), 606-613.

Kruschke, J. K. (2018). Rejecting or accepting parameter values in Bayesian estimation. Advances in Methods and Practices in Psychological Science, 1(2), 270-280.

Lakens, D., Scheel, A. M., \& Isager, P. M. (2018). Equivalence testing for psychological research: A tutorial. Advances in Methods and Practices in Psychological Science, 1(2), 259-269.

Langkaas, T. F., Wampold, B. E., \& Hoffart, A. (2018). Five types of clinical difference to monitor in practice. Psychotherapy, 55(3), 241.

Lassere, M., van der Heijde, D., \& Johnson, K. R. (2001). Foundations of the minimal clinically important difference for imaging. The Journal of Rheumatology, 28(4), 890-891.

Maassen, G. H. (2000). Principles of defining reliable change indices. Journal of Clinical and Experimental Neuropsychology, 22(5), 622-632.

Maassen, G. H. (2001). The unreliable change of reliable change indices. Behaviour Research and Therapy, 39(4), 495-498.

Maassen, G. H. (2004). The standard error in the Jacobson and Truax Reliable Change Index (the 
classical approach to the assessment of reliable change). Journal of the International Neuropsychological Society, 10, 888-893.

Maassen, G. H., Bossema, E., \& Brand, N. (2009). Reliable change and practice effects: Outcomes of various indices compared. Journal of Clinical and Experimental Neuropsychology, 31(3), 339352.

Martinovich, Z., Saunders, S., \& Howard, K. (1996). Some comments on "Assessing clinical significance." Psychotherapy Research, 6(2), 124-132.

Marx, B. P., Lee, D. J., Norman, S. B., Bovin, M. J., Sloan, D. M., Weathers, F. W., Keane, T. M., \& Schnurr, P. P. (2021). Reliable and clinically significant change in the clinician-administered PTSD Scale for DSM-5 and PTSD Checklist for DSM-5 among male veterans. Psychological Assessment.

McAleavey, A. A. (2022a). One in five, not one in 17, youth patients deteriorate during psychotherapy for depression. European Child \& Adolescent Psychiatry, 1-4.

McAleavey, A. A. (2022b). ReliableTrendIndex: Utilities for (More Reliable) Reliable Change Analysis. https://github.com/andrewmcaleavey/ReliableTrendIndex

McAleavey, A. A., Youn, S. J., Xiao, H., Castonguay, L. G., Hayes, J. A., \& Locke, B. D. (2019). Effectiveness of routine psychotherapy: Method matters. Psychotherapy Research, 29(2), 139156. https://doi.org/10.1080/10503307.2017.1395921

McCrae, R. R., Kurtz, J. E., Yamagata, S., \& Terracciano, A. (2011). Internal consistency, retest reliability, and their implications for personality scale validity. Personality and Social Psychology Review, 15(1), 28-50.

McNemar, Q. (1958). On growth measurement. Educational and Psychological Measurement, 18(1), 4755.

Molenaar, P. C. M. (2004). A Manifesto on Psychology as Idiographic Science: Bringing the Person Back Into Scientific Psychology, This Time Forever. Measurement: Interdisciplinary Research and Perspectives, 2(4), 201-218. https://doi.org/10.1207/s15366359mea0204_1 
Morgan-Lopez, A. A., Saavedra, L. M., Ramirez, D. D., Smith, L. M., \& Yaros, A. C. (2022). Adapting the multilevel model for estimation of the reliable change index (RCI) with multiple timepoints and multiple sources of error. International Journal of Methods in Psychiatric Research, e1906.

Peipert, J. D., Hays, R. D., \& Cella, D. (2022). Likely change indexes improve estimates of individual change on patient-reported outcomes. Quality of Life Research, 1-12.

Pilkonis, P. A., Yu, L., Dodds, N. E., Johnston, K. L., Maihoefer, C. C., \& Lawrence, S. M. (2014). Validation of the depression item bank from the Patient-Reported Outcomes Measurement Information System (PROMIS $\left.{ }^{\circledR}\right)$ in a three-month observational study. Journal of Psychiatric Research, 56, 112-119.

Radloff, L. S. (1977). The CES-D scale: A self-report depression scale for research in the general population. Applied Psychological Measurement, 1(3), 385-401.

Revelle, W., \& Condon, D. M. (2019). Reliability from $\alpha$ to \$ømega\$: A tutorial. Psychological Assessment, 31(12), 1395.

Revicki, D., Hays, R. D., Cella, D., \& Sloan, J. (2008). Recommended methods for determining responsiveness and minimally important differences for patient-reported outcomes. Journal of Clinical Epidemiology, 61(2), 102-109.

Robinaugh, D. J., Brown, M. L., Losiewicz, O. M., Jones, P. J., Marques, L., \& Baker, A. W. (2020). Towards a precision psychiatry approach to anxiety disorders with ecological momentary assessment: The example of panic disorder. General Psychiatry, 33(1).

Ronk, F. R., Hooke, G. R., \& Page, A. C. (2016). Validity of clinically significant change classifications yielded by Jacobson-Truax and Hageman-Arrindell methods. BMC Psychiatry, 16(1), 1-9.

Royston, P., Altman, D. G., \& Sauerbrei, W. (2006). Dichotomizing continuous predictors in multiple regression: A bad idea. Statistics in Medicine, 25(1), 127-141.

Sijtsma, K. (2009). On the use, the misuse, and the very limited usefulness of Cronbach's alpha. Psychometrika, 74(1), 107.

Snapinn, S. M., \& Jiang, Q. (2007). Responder analyses and the assessment of a clinically relevant 
treatment effect. Trials, 8(1), 1-6.

Speer, D. C. (1999). What is the role of two-wave designs in clinical research? Comment on Hageman and Arrindell. Behaviour Research and Therapy, 37(12), 1203-1210. https://doi.org/10.1016/S0005-7967(99)00034-0

Speer, D. C., \& Greenbaum, P. E. (2002). Correction to Speer and Greenbaum (1995). Journal of Consulting and Clinical Psychology, 70(6), 1239. https://doi.org/10.1037/0022-006X.70.6.1239

Stefanski, L. A. (2000). Measurement error models. Journal of the American Statistical Association, 95(452), 1353-1358.

Temkin, N. R., Heaton, R. K., Grant, I., \& Dikmen, S. S. (1999). Detecting significant change in neuropsychological test performance: A comparison of four models. Journal of the International Neuropsychological Society, 5(4), 357-369.

Terwee, C. B., Roorda, L. D., Knol, D. L., De Boer, M. R., \& De Vet, H. C. (2009). Linking measurement error to minimal important change of patient-reported outcomes. Journal of Clinical Epidemiology, 62(10), 1062-1067.

Thissen, D., Liu, Y., Magnus, B., Quinn, H., Gipson, D. S., Dampier, C., Huang, I.-C., Hinds, P. S., Selewski, D. T., Reeve, B. B., \& others. (2016). Estimating minimally important difference (MID) in PROMIS pediatric measures using the scale-judgment method. Quality of Life Research, 25(1), 13-23.

Trafimow, D. (2014). Estimating true standard deviations. Frontiers in Psychology, 5, 235. https://doi.org/10.3389/fpsyg.2014.00235

Trajković, G., Starčević, V., Latas, M., Leštarević, M., Ille, T., Bukumirić, Z., \& Marinković, J. (2011). Reliability of the Hamilton Rating Scale for Depression: A meta-analysis over a period of 49 years. Psychiatry Research, 189(1), 1-9.

Turner, D., Schünemann, H. J., Griffith, L. E., Beaton, D. E., Griffiths, A. M., Critch, J. N., \& Guyatt, G. H. (2010). The minimal detectable change cannot reliably replace the minimal important difference. Journal of Clinical Epidemiology, 63(1), 28-36. 
Vasishth, S., Mertzen, D., Jäger, L. A., \& Gelman, A. (2018). The statistical significance filter leads to overoptimistic expectations of replicability. Journal of Memory and Language, 103, 151-175.

Viswanathan, M. (2005). What causes measurement error? In Measurement Error and Research Design (pp. 135-148). Sage.

Wallis, P. S. (2013). The impact of screen format and repeated assessment on responses to a measure of depressive symptomology completed twice in a short timeframe [PhD Thesis]. Arts \& Social Sciences: Department of Psychology.

Watson, D. (2004). Stability versus change, dependability versus error: Issues in the assessment of personality over time. Journal of Research in Personality, 38(4), 319-350.

Wise, E. A. (2004). Methods for analyzing psychotherapy outcomes: A review of clinical significance, reliable change, and recommendations for future directions. Journal of Personality Assessment, $82(1), 50-59$.

Wolpert, M., Görzig, A., Deighton, J., Fugard, A. J., Newman, R., \& Ford, T. (2015). Comparison of indices of clinically meaningful change in child and adolescent mental health services: Difference scores, reliable change, crossing clinical thresholds and 'added value'-an exploration using parent rated scores on the SDQ. Child and Adolescent Mental Health, 20(2), 94-101.

Youngstrom, E. A., Van Meter, A., Frazier, T. W., Hunsley, J., Prinstein, M. J., Ong, M.-L., \& Youngstrom, J. K. (2017). Evidence-based assessment as an integrative model for applying psychological science to guide the voyage of treatment. Clinical Psychology: Science and Practice, 24(4), 331-363. 
Figure 1. Suggested flowchart when considering using the RCI.
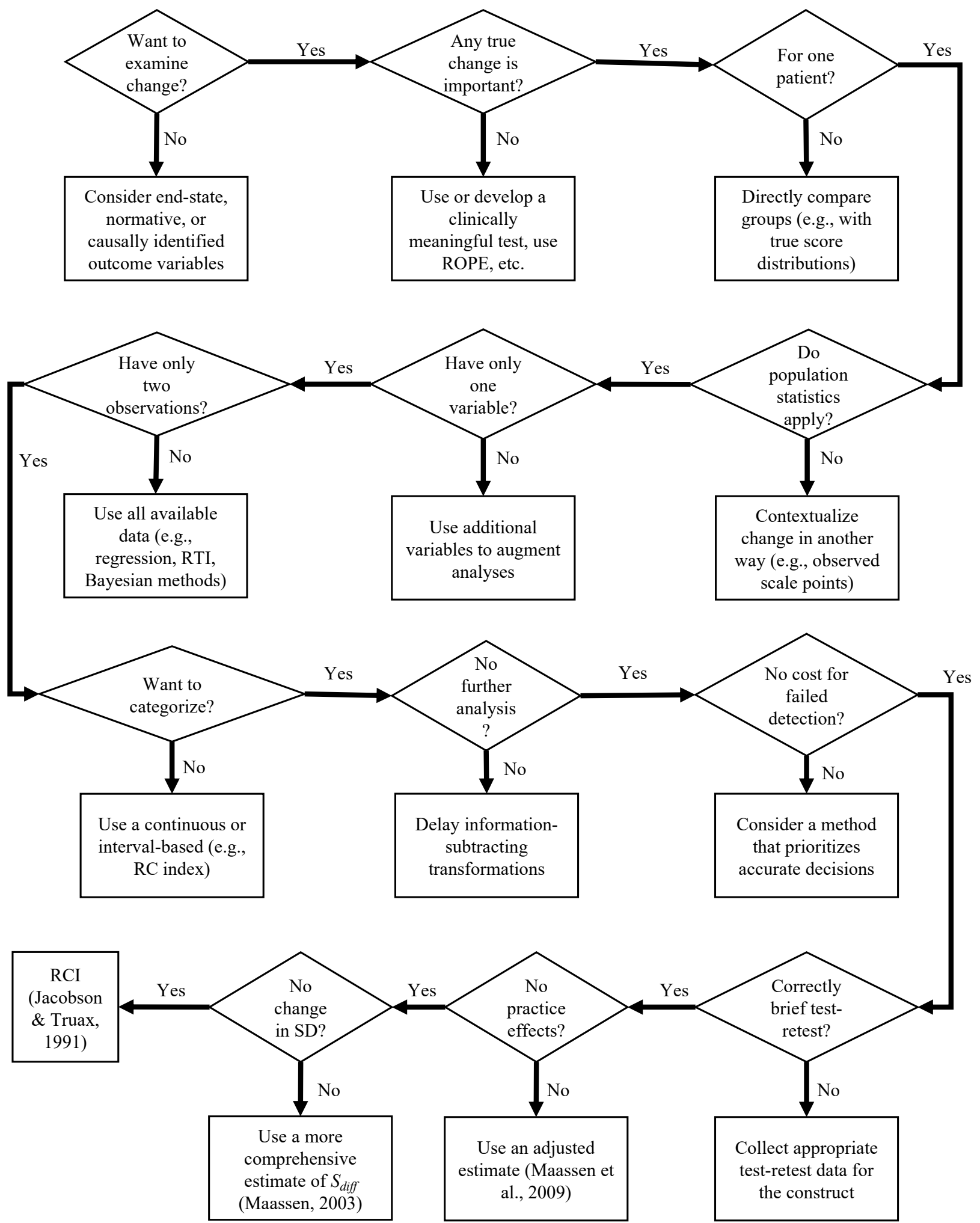
Figure 2. Total accuracy of the RCI and the sign of the difference as a function of true change.

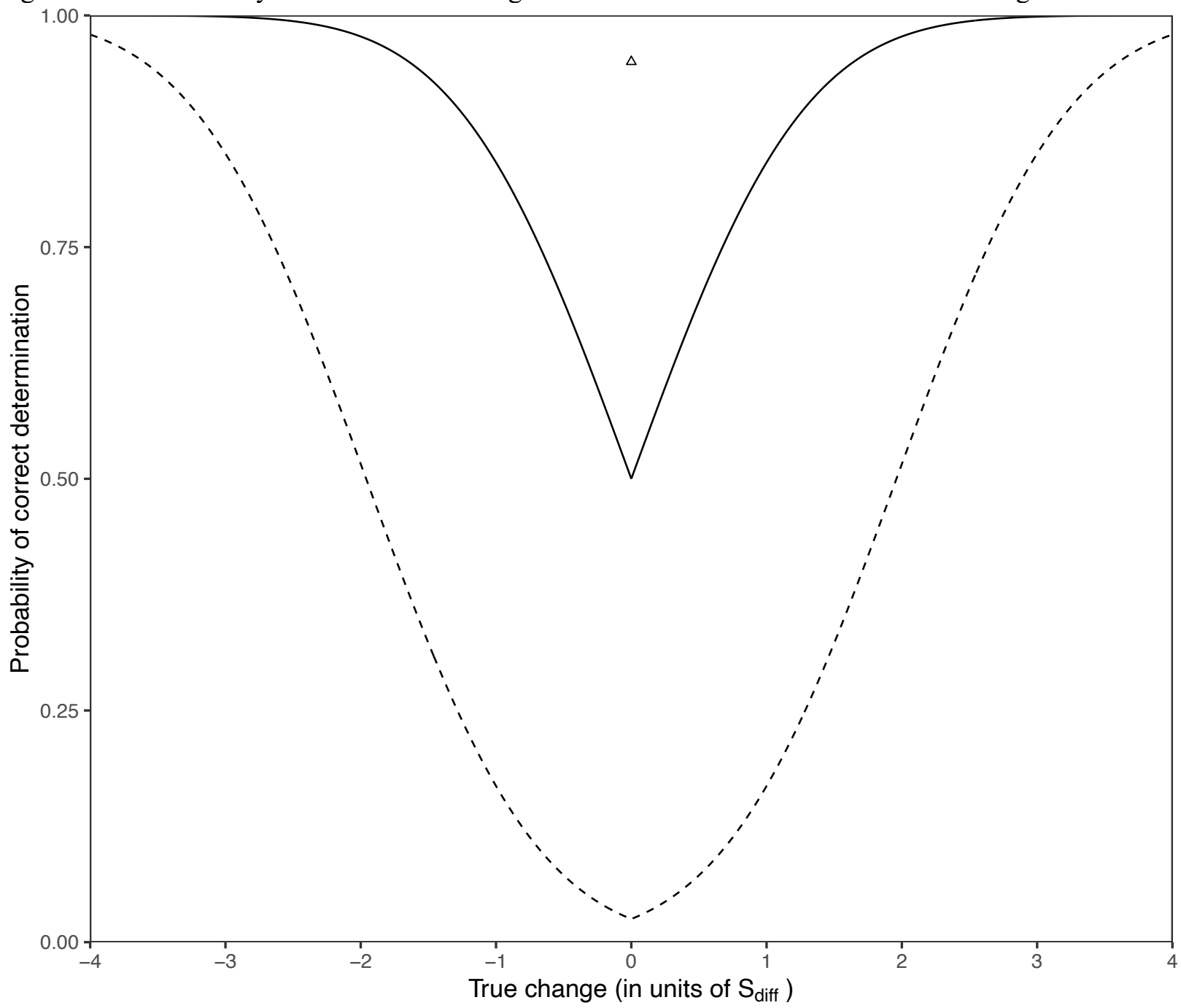

Note. The solid line is the total accuracy of the sign of the observed difference score while the dashed line and the triangle is the total accuracy of the RCI as defined in Eq. 2. Except when the true change is exactly 0 , the RCI will always be less accurate than the sign of the difference score, often considerably so, because the RCI frequently fails to detect real change. This plot assumes an ideal continuous measure and perfect satisfaction of the RCI's assumptions. The x-axis is also interpretable as the SNR. 
Figure 3. Measurement errors: the RCI compared to the sign of the difference score.

A

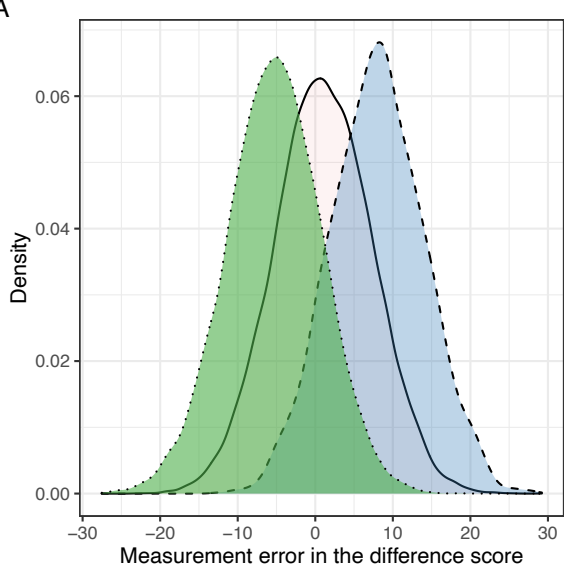

C

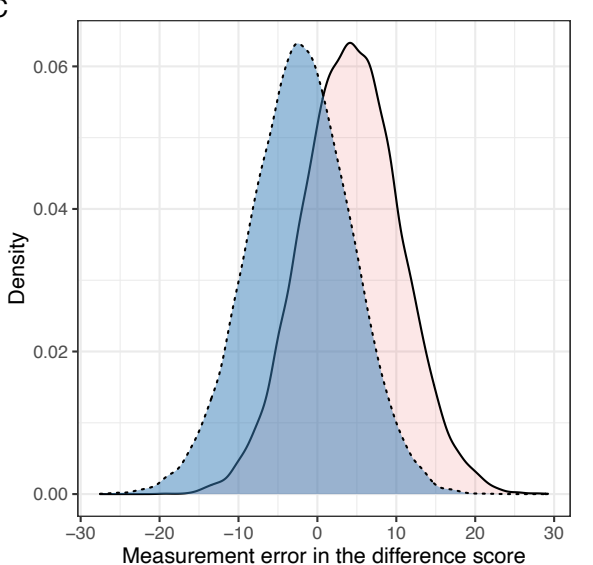

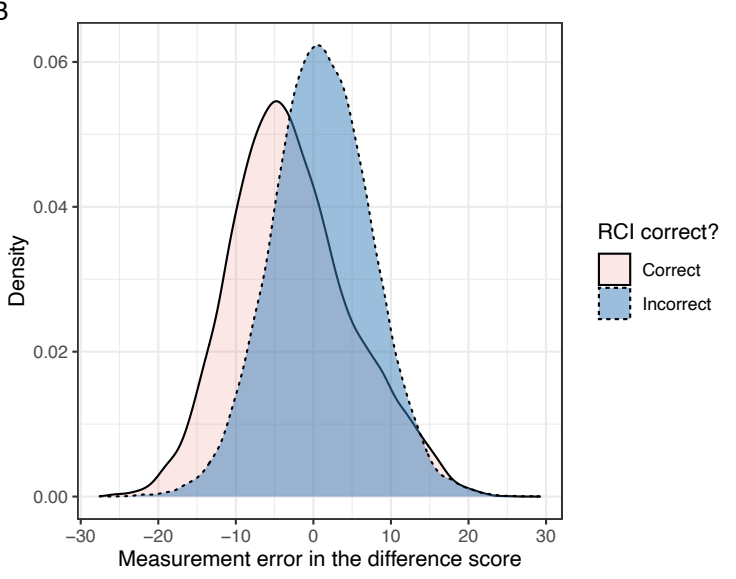

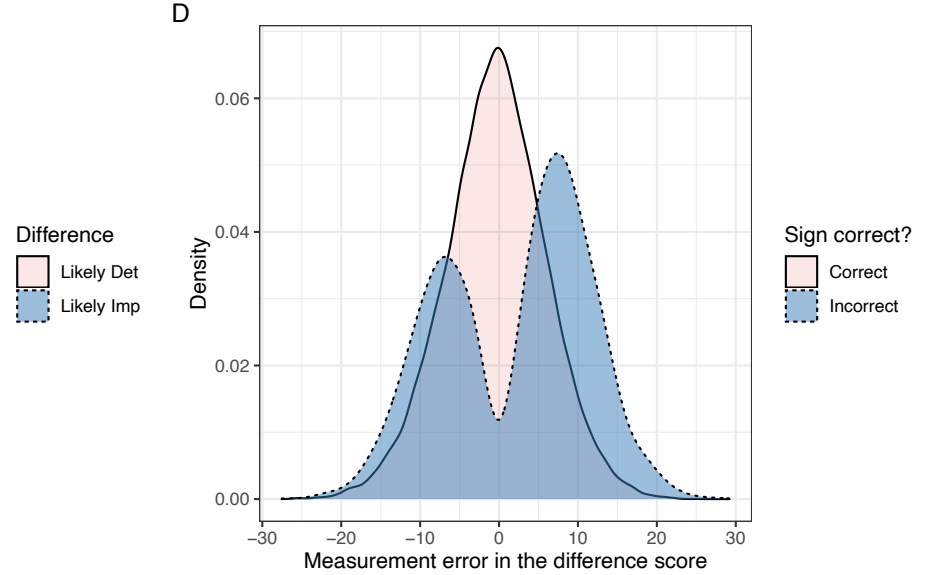

Note. Cutpoints induce biased errors, so "reliable" changes are often inaccurate and biased. This is simulated data $(N=50,000)$ with average improvement $(d=-.45)$ true change score heterogeneity, and a typically reliable instrument (test-retest reliability $r=.80)$. Panel A: Individuals who are categorized as reliably changed have biased and large measurement errors, while those whose change scores are smaller than the RCI have unbiased and smaller average errors. Panel B: When the RCI makes the correct decision in this data, measurement error is biased and negative (this is the source of the exaggeration effect), while when it was incorrect the errors are relatively unbiased, meaning the RCI is less accurate with accurate measurements. Panel C: Relying on the difference score alone as a decision cutpoint also induces some bias in errors, though less than the RCI categories in Panel A. Panel D: Unlike Panel B above, when the difference score is correct, errors are small and unbiased, and when it is incorrect, errors are relatively large. Complete simulation details are available in the Supplement. 